\title{
Distinct VEGF Functions During Bone Development and Homeostasis
}

\author{
Yanqiu Liu • Bjorn R. Olsen
}

Received: 7 October 2013 / Accepted: 18 February 2014/Published online: 4 April 2014

(C) L. Hirszfeld Institute of Immunology and Experimental Therapy, Wroclaw, Poland 2014

\begin{abstract}
Vascular endothelial growth factor-A (VEGF) is a key regulator of physiological hemangiogenesis during development, postnatal growth, and homeostasis. It is well known that VEGF is required for effective coupling of angiogenesis to endochondral and membranous bone formation during skeletal development. However, less well known are the roles of VEGF in regulating the differentiation and/or functions of skeletal cells such as chondrocytes, osteoblasts, and osteoclasts. In this review, we discuss some of these functions. During early skeletal development, VEGF is important for the survival of chondrocytes in the hypoxic regions of the cartilage models of future bones, the vascularization of developing bones and proliferation and differentiation of osteoblastic cells. Postnatally, osteoblast-derived VEGF is critical for maintaining bone homeostasis by stimulating the differentiation of mesenchymal stem cells to osteoblasts and repressing their differentiation to adipocytes. Recent data indicate that these effects of VEGF on osteogenic/adipogenic stem cell fates are based on an intracellular (intracrine) mechanism. In contrast, osteoblast-derived VEGF is also known to stimulate the differentiation of monocytes to osteoclasts by a paracrine mechanism. Mice with VEGF-deficient osteoblastic lineage cells exhibit age-dependent loss of bone mass and an increase in bone marrow fat. These changes are similar to the changes associated with osteoporosis in
\end{abstract}

Y. Liu · B. R. Olsen $(\bowtie)$

Department of Developmental Biology, Harvard School of Dental Medicine, 188 Longwood Ave., Boston, MA 02115, USA

e-mail: bjorn_olsen@hms.harvard.edu

Present Address:

Y. Liu ( $\square)$

399 Margarita Ave., Palo Alto, CA 94306, USA

e-mail: yanqiu_liu@post.harvard.edu humans. Thus, a better understanding of the intracellular mechanisms by which VEGF regulates osteoblastic and adipogenic differentiation may lead to the identification of new targets for therapies to prevent osteoporotic bone loss.

Keywords VEGF · Mesenchymal stem cells ·

Ossification · Osteoblast · Adipocyte - Differentiation

\section{VEGF and Its Receptors}

Vascular endothelial growth factor-A (VEGF) is a key regulator of physiological hemangiogenesis during embryonic development and postnatal growth and homeostasis. VEGF enhances endothelial cell proliferation, migration and survival and promotes vascular permeability (Ferrara et al. 2003). The human VEGFA gene is organized as eight exons separated by seven introns. Alternative exon splicing results in the generation of six different isoforms named according to the number of amino acid residues after signal peptide cleavage (Conn et al. 1990; Pepper et al. 1994). VEGF165 (human)/VEGF164 (mouse), the predominant isoform, lacks the heparin-binding domain encoded by exon 6, whereas VEGF121 (human)/VEGF120 (mouse) lacks the heparin-binding domains encoded by exons 6 and 7 (Pepper et al. 1992). VEGF189 and VEGF206 are highly basic and bind to heparin with high affinity (Nicosia et al. 1994).

In vascular endothelial cells, VEGF primarily binds to two tyrosine kinase receptors, VEGFR-1 and VEGFR-2. In addition, endothelial cells express the co-receptor neuropilin 1 (NRP1), which binds to VEGF165 and potentiates VEGFR-2 activity (Soker et al. 1998). VEGFR-2 (or fetal liver kinase-1: Flk-1) is a major mediator of the mitogenic, angiogenic, and permeability-enhancing effects of VEGF 
(Ferrara et al. 2003). The key role of this receptor in developmental angiogenesis and hematopoiesis is evident by the lack of vasculogenesis and failure to develop blood islands and organized blood vessels in Flk1-null mice, resulting in embryonic lethality between embryonic (E) days E8.5 and E9.5 (Shalaby et al. 1995). VEGFR-1 (or fms-like tyrosine kinase-1: Flt-1) has minimal kinase activity although it binds to VEGF with high affinity. Both a membrane-bound form of VEGFR-1 and a soluble form, generated by alternative splicing of the Flt-1 gene, are "decoy" receptors that are able to negatively regulate the activity of VEGF on the vascular endothelium by preventing VEGF binding to VEGFR-2 (Park et al. 1994). Homozygous Flt-1 null mice die in utero between E8.5 and E9.5 due to vascular disorganization caused by over growth of endothelial cells, indicating that VEGFR-1 is indeed a negative regulator of VEGF action (Fong et al. 1999).

\section{The Role of VEGF in Skeletal Development}

In contrast to VEGF signaling in endothelial cells that has been widely studied, VEGF signaling in osteoblasts remains less well known. Osteoblasts express VEGF receptors (VEGFR-1, VEGFR-2) and the VEGF binding co-receptor neuropilin (Deckers et al. 2000; Harper et al. 2001). Expression of VEGF and its receptors in differentiating osteoblasts has been detected in cultured cells (Deckers et al. 2000), and in vivo by in situ hybridization (Zelzer et al. 2002). In vitro cell culture studies suggested that VEGFR-2 plays a role in both osteoblast differentiation and survival (Alonso et al. 2008).

In vertebrate development, there are two types of bone formation: intramembranous bone formation and endochondral bone formation. Bone formation begins when mesenchymal cells form condensations in regions of the future bones. In intramembranous bone formation, as in the flat bones of the skull, the cells of these condensations differentiate into bone-forming osteoblasts and lay down an extracellular matrix (osteoid) rich in type I collagen. In endochondral bone formation, the cells of the condensations differentiate to chondrocytes and form cartilage models of the future bones. As the cartilage models grow through chondrocyte proliferation and extracellular matrix production, the chondrocytes in their central regions stop proliferating, enlarge (hypertrophy), and synthesize a type $\mathrm{X}$ collagen-rich matrix. The hypertrophic chondrocytes direct adjacent perichondrial cells to become osteoblasts and form a bone collar around the central region of the developing bone. They also express high levels of VEGF. This acts as a chemotactic factor for invasion of osteoclasts, vascular endothelial cells, and hematopoietic progenitors into the hypertrophic cartilage from the surrounding perichondrium/periosteum. The cartilage matrix left behind provides a scaffold for osteoblastic progenitor cell that invades the cartilage along with sprouting blood vessels and lay down a mineralizing trabecular bone matrix (Karsenty 2003; Kronenberg 2003; Zelzer and Olsen 2003). In mice that express only the VEGF120 isoform, recruitment of blood vessels into the perichondrium and the invasion of angiogenic sprouts, osteoclasts and osteoblastic and hematopoietic progenitors into the primary ossification center is severely delayed, and bone mineralization is reduced (Zelzer et al. 2002). Inhibition of VEGF by administration of soluble chimeric VEGFR protein to 24-day-old mice inhibited blood vessel invasion into the hypertrophic zone of long bone growth plates and resulted in impaired trabecular bone formation and expansion of the hypertrophic zone (Gerber et al. 1999).

In addition to being required for bone vascularization, VEGF is also a critical factor for survival of chondrocytes. The epiphyseal regions of some long bones in mice expressing only the VEGF188 isoform have been reported to contain areas of chondrocytic cell death (Maes et al. 2004). In mice with VEGF deficiency in chondrocytes (produced by crossing mice carrying floxed VEGF alleles with Col2 $\alpha 1$-Cre mice), chondrocytes have been found to undergo massive cell death in the central regions of the skeletal elements, starting at an articular surface and continuing through the resting to the proliferating zone of growth plate chondrocytes, resulting in a misshapen growth plate (Zelzer et al. 2004). A similar phenotype has been described in epiphyseal cartilage of mice in which the transcription factor HIF-1 $\alpha$ is conditionally inactivated in chondrocytes (Schipani et al. 2001). This suggests that VEGF and HIF-1 $\alpha$ are part of a chondrocyte survival pathway. VEGF appears to be downstream of HIF-1 $\alpha$ in chondrocyte survival since VEGF expression in epiphyseal cells depends on HIF-1 $\alpha$ (Schipani et al. 2001; Zelzer et al. 2004).

\section{The Role of VEGF in Osteoblast Function}

Several factors with important roles in regulating bone formation induce the expression of VEGF by osteoblasts, such as BMP, FGF, TGF- $\beta$, IGF, and vitamin D3 (Zelzer and Olsen 2005). This raises the question of what role osteoblast-derived VEGF plays in osteoblast function. In support of an important role are data from studies of mice that only express VEGF120. In these mice, mineralization and expression of osteoblastic markers of intramembranous bones were reduced (Zelzer et al. 2002). Furthermore, VEGF induces differentiation of cultured osteoblasts (Deckers et al. 2000; Midy and Plouet 1994) and stimulates 
chemotactic migration and proliferation of primary human osteoblasts (Mayr-Wohlfart et al. 2002).

These findings suggest that VEGF may regulate bone formation through an effect on osteoblasts in addition to its role in coupling angiogenesis and endochondral bone formation, but evidence for a role of osteoblast-derived VEGF in bone development has only recently been obtained. This evidence indicates that VEGF produced by osteoblastic lineage cells helps to maintain postnatal bone homeostasis by promoting osteoblast differentiation and inhibiting adipocyte differentiation. Mice with conditional VEGF deficiency in osteoblastic progenitor cells exhibit a progressive reduction in bone mass and an increase in bone marrow fat, starting about 3 weeks after birth (Liu et al. 2012). Interestingly, these changes are similar to changes that are associated with age-dependent osteoporosis. The decrease in bone volume associated with osteoporosis and age-related osteopenia is also accompanied by an increase in marrow adipose tissue (Verma et al. 2002). Marrow adipocytes share a common mesenchymal stem cell (MSC) with bone-forming osteoblasts and a large degree of plasticity exists between the osteoblastic and adipocytic lineages (Beresford et al. 1992; Nuttall et al. 1998; Pittenger et al. 1999). Human bone marrow-derived MSCs have multi-lineage differentiation potential and fully differentiated osteoblasts derived from human MSCs have been reported to be capable of trans-differentiation into adipocytes and chondrocytes, and vice versa (Song and Tuan 2004). This plasticity has important therapeutic implications. Understanding the factors and pathways that control osteoblastic and adipocytic differentiation should be of relevance to the development of therapies to control bone loss in osteoporosis (Song and Tuan 2004).

The reduction in bone mass and the increase in bone marrow fat in VEGF conditional knockout mice (VEGF is specifically deleted in osteoblast lineage cells) (Liu et al. 2012) raises the question of whether VEGF or VEGF signaling would be a target for therapy to treat osteoporosis. Interestingly, postmenopausal women with polymorphisms associated with high or low VEGF production have been reported to have higher or lower lumbar spine bone mineral density, respectively (Costa et al. 2009). However, there appears to be no significant association between levels of circulating VEGF and bone mineral density (Costa et al. 2009). A possible explanation for these observations is provided by the findings that VEGF appears to stimulate osteoblastic differentiation and suppress adipocytic differentiation by an intracellular mechanism rather than by paracrine signaling (Liu et al. 2012). Treatment with recombinant VEGF or neutralizing antibodies against VEGF does not affect osteoblastic and adipocytic differentiation in bone marrow-derived MSC cultures. Also, addition of recombinant VEGF to cultures of VEGF- deficient MSCs, even at very high concentrations, has no effect on the differentiation properties of the mutant cells (Liu et al. 2012). However, virus-mediated expression of VEGF in VEGF-deficient MSCs normalizes both osteoblast and adipocyte differentiation. This suggests that VEGF controls osteoblastogenesis and adipogenesis via mechanisms that are resistant to the effects of extracellular VEGF or antibodies against VEGF.

The concept of an intracrine signaling loop for VEGF has been previously suggested in the case of bone marrow hematopoietic stem cells (HSC) (Gerber et al. 2002). A reduction in survival, colony formation, and in vivo repopulation rates of HSCs was observed after ablation of the VEGF gene in mice. Intracellularly acting small-molecule inhibitors of VEGFR tyrosine kinase dramatically reduced colony formation of HSCs, thus mimicking deletion of the VEGF gene. However, blocking VEGF activity by administering a soluble form of VEGFR-1, which acts extracellularly, induced only minor effects. These findings support the involvement of VEGF in HSC survival by an internal autocrine loop mechanism (that is, the mechanism is resistant to inhibitors that fail to penetrate the intracellular compartment). A similar phenomenon has been reported in studies in which VEGF was specifically deleted in endothelial cells (Lee et al. 2007). This led to loss of postnatal endothelial cell survival but this loss could not be prevented by exogenous VEGF.

When the VEGF receptors Flt- 1 and Flk- 1 were conditionally targeted in osteoblastic lineage cells in mice (Liu et al. 2012), examination of mutant skeletons suggested that both receptors may be required for the stimulatory effect of VEGF on osteoblastogenesis, but they are not critical for the ability of VEGF to repress adipocyte differentiation. Consistent with a role for VEGF receptors in osteoblast differentiation is the finding that bones of mice lacking the Flt- 1 intracellular tyrosine kinase domain have reduced trabecular bone volume (Otomo et al. 2007). How VEGFR-2 affects osteoblast development is not clear, but in vitro cell culture studies indicated that VEGFR-2 plays a role in osteoblast differentiation and survival (Alonso et al. 2008).

A large body of conflicting evidence exists in the studies of VEGFR expression by osteoblasts, depending on the use of different osteoblastic cells from different species and the use of different methods to detect VEGFR expression. Expression of VEGFR-1 and VEGFR-2 in addition to NRP1 has been reported in a murine preosteoblast cell line (KS483) using RT-PCR (Deckers et al. 2000) and VEGFR1 expression in osteoblasts was reported using in situ hybridization (Gerber et al. 1999). In contrast, Harper et al. (2001) found that MC3T3-E1 osteoblasts do not express VEGFR-1 and VEGFR-2 using Northern blotting for detection. Instead, NRP1 was found to be expressed in 
these cells and could be cross-linked with VEGF165, suggesting that NRP1 is a major VEGFR in MC3T3-E1 osteoblasts. Further studies using primary human and rat long bone-derived osteoblast-like cells suggested that only weak ERK 1/2 activation and Cox-2 induction could be elicited in response to exogenous VEGF although VEGFR2 expression was detectable by immunofluorescence (Clarkin et al. 2008), raising the possibility that intracrine VEGF signaling may play a role in these cells. Expression levels of VEGF and VEGF receptors in osteoblasts is also regulated by mechanotransduction and MicroRNAs (Gao et al. 2011; Thi et al. 2010).

In addition to intracrine mechanisms, it is possible that osteoblast-derived VEGF also affects bone formation through enhanced angiogenesis since it has been reported that HIF- $1 \alpha$ promotes angiogenesis and osteogenesis by elevating VEGF levels in osteoblasts (Wang et al. 2007). During bone regeneration induced by the technique of distraction osteogenesis, immunohistological and qRTPCR analysis showed that VEGFR-2 appeared to be predominantly expressed by vascular endothelial and hematopoietic cells (Matsubara et al. 2012). It will be interesting to investigate whether and how osteoblastderived VEGF affects the interaction between osteoblasts and endothelial cells in bone marrow since it has been reported that direct contact between endothelial cells and osteoprogenitors supports osteoblast function in vitro (Guillotin et al. 2008).

\section{Osteoblast-Derived VEGF Stimulates Osteoclast Formation}

Osteoclasts are terminally differentiated cells derived from the monocyte/macrophage lineage and serve a critical function in bone resorption. The differentiation, activation, and survival of osteoclasts are primarily regulated by colony-stimulating factor 1/macrophage colony-stimulating factor (M-CSF) and RANK (receptor activator of nuclear factor of NF- $\mathrm{kB}$ ) ligand (RANKL) (Tanaka et al. 1993).

The op/op mouse (homozygous for the recessive osteopetrosis mutation) exhibits a severe deficiency of osteoclasts, monocytes, and tissue macrophages due to lack of M-CSF function (Kodama et al. 1991). VEGF can substitute for M-CSF in osteoclast formation in op/op mice since administration of VEGF ameliorated osteoclastogenesis and bone resorption and a VEGF antagonist suppressed the increase in osteoclast formation that occurs in op/op mice with age (Niida et al. 1999). Bone marrow cell culture experiments demonstrate that VEGF can substitute for M-CSF and cooperate with RANKL to support osteoclast differentiation (Niida et al. 1999). Furthermore, VEGF can directly enhance osteoclastic bone resorption and survival of mature osteoclasts via VEGFR-2 signaling (Nakagawa et al. 2000; Yang et al. 2008). In our recent studies of osteoblast-derived VEGF (Liu et al. 2012), we confirmed that VEGF is a stimulator of osteoclastogenesis. However, in contrast to the intracellular mechanism by which osteoblast-derived VEGF stimulates osteoblastic differentiation, this effect of osteoblast-derived VEGF on osteoclast formation is based on a paracrine mechanism. Consistent with such a paracrine effect of VEGF on osteoclast formation is our finding that osteoclast formation with bone marrow cells from mice lacking VEGFR-1 in osteoblastic cells is dramatically increased compared to control mice. Since VEGFR-1 can act as a decoy receptor for VEGF (Park et al. 1994), this increased osteoclast formation in the mutant cultures may be the result of excessive amounts of VEGF available for signaling through VEGFR-2 receptors on osteoclast progenitor cells.

\section{Summary and Future Directions}

VEGF plays an important role in skeletal development. Postnatally, osteoblast-derived VEGF regulates the balance
Fig. 1 VEGF regulates bone homeostasis via both intracrine and paracrine functions. VEGF stimulates osteoblast differentiation and inhibits adipocyte differentiation via intracrine function. Osteoblastderived VEGF exerts paracrine function on osteoclast differentiation

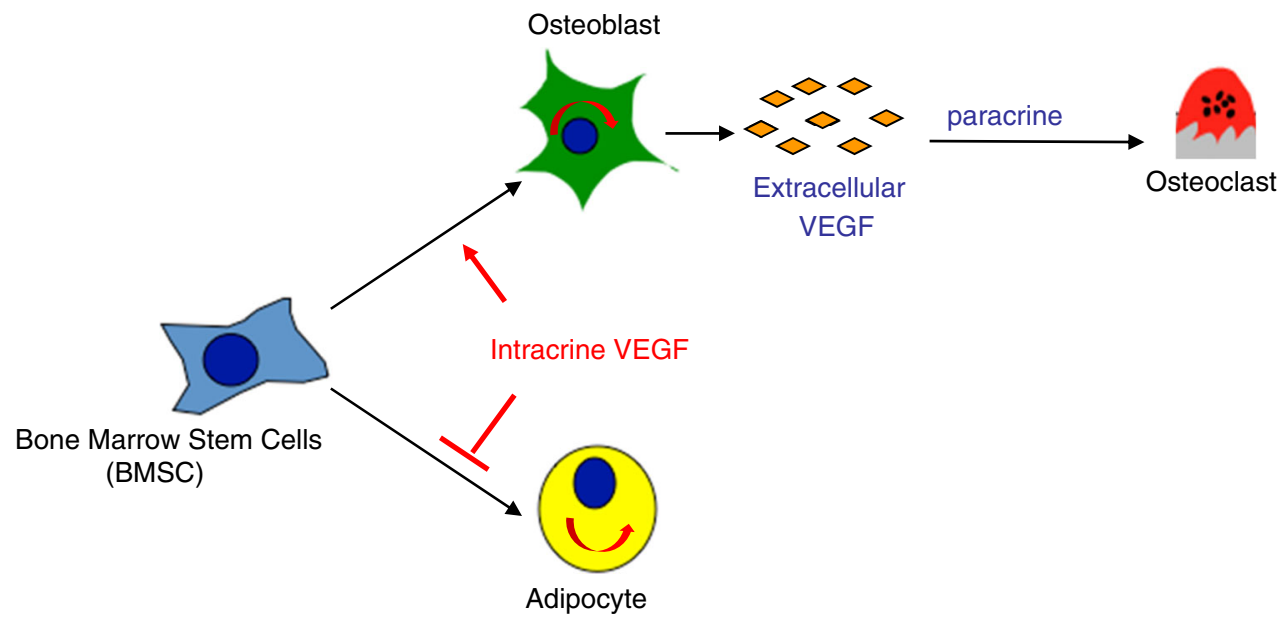


between osteoblastogenesis and adipogenesis in bone marrow and it stimulates osteoclast differentiation (Fig. 1). VEGF appears to control the differentiation of MSCs by stimulating the transcription factor RUNX2 and repressing the transcription factor PPAR $\gamma 2$ (Liu et al. 2012). Interestingly, the data also suggest that there is a functional interaction between VEGF and the nuclear envelope protein lamin $\mathrm{A} / \mathrm{C}$, but further investigations are needed to elucidate the detailed mechanisms by which intracrine VEGF signaling controls osteoblast and adipocyte differentiation in MSCs. Fully understanding these intracrine VEGF signaling pathways will likely contribute to the identification of novel targets for therapies to control bone loss in osteoporosis (Prockop 2012).

Conflict of interest The authors have declared that no conflict of interest exists.

\section{References}

Alonso V, de Gortazar AR, Ardura JA et al (2008) Parathyroid hormone-related protein (107-139) increases human osteoblastic cell survival by activation of vascular endothelial growth factor receptor-2. J Cell Physiol 217:717-727

Beresford JN, Bennett JH, Devlin C et al (1992) Evidence for an inverse relationship between the differentiation of adipocytic and osteogenic cells in rat marrow stromal cell cultures. J Cell Sci 102(Pt 2):341-351

Clarkin CE, Garonna E, Pitsillides AA et al (2008) Heterotypic contact reveals a COX-2-mediated suppression of osteoblast differentiation by endothelial cells: a negative modulatory role for prostanoids in VEGF-mediated cell: cell communication? Exp Cell Res 314:3152-3161

Conn G, Bayne ML, Soderman DD et al (1990) Amino acid and cDNA sequences of a vascular endothelial cell mitogen that is homologous to platelet-derived growth factor. Proc Natl Acad Sci USA 87:2628-2632

Costa N, Paramanathan S, Mac Donald D et al (2009) Factors regulating circulating vascular endothelial growth factor (VEGF): association with bone mineral density (BMD) in postmenopausal osteoporosis. Cytokine 46:376-381

Deckers MM, Karperien M, van der Bent C et al (2000) Expression of vascular endothelial growth factors and their receptors during osteoblast differentiation. Endocrinology 141:1667-1674

Ferrara N, Gerber HP, LeCouter J (2003) The biology of VEGF and its receptors. Nat Med 9:669-676

Fong GH, Zhang L, Bryce DM et al (1999) Increased hemangioblast commitment, not vascular disorganization, is the primary defect in flt-1 knock-out mice. Development 126:3015-3025

Gao J, Yang T, Han J et al (2011) MicroRNA expression during osteogenic differentiation of human multipotent mesenchymal stromal cells from bone marrow. J Cell Biochem 112:1844-1856

Gerber HP, Vu TH, Ryan AM et al (1999) VEGF couples hypertrophic cartilage remodeling, ossification and angiogenesis during endochondral bone formation. Nat Med 5:623-628

Gerber HP, Malik AK, Solar GP et al (2002) VEGF regulates haematopoietic stem cell survival by an internal autocrine loop mechanism. Nature 417:954-958

Guillotin B, Bareille R, Bourget C et al (2008) Interaction between human umbilical vein endothelial cells and human osteoprogenitors triggers pleiotropic effect that may support osteoblastic function. Bone 42:1080-1091

Harper J, Gerstenfeld LC, Klagsbrun M (2001) Neuropilin-1 expression in osteogenic cells: down-regulation during differentiation of osteoblasts into osteocytes. J Cell Biochem 81:82-92

Karsenty G (2003) The complexities of skeletal biology. Nature 423:316-318

Kodama H, Yamasaki A, Nose M et al (1991) Congenital osteoclast deficiency in osteopetrotic (op/op) mice is cured by injections of macrophage colony-stimulating factor. J Exp Med 173:269-272

Kronenberg HM (2003) Developmental regulation of the growth plate. Nature 423:332-336

Lee S, Chen TT, Barber CL et al (2007) Autocrine VEGF signaling is required for vascular homeostasis. Cell 130:691-703

Liu Y, Berendsen AD, Jia S et al (2012) Intracellular VEGF regulates the balance between osteoblast and adipocyte differentiation. J Clin Invest 122:3101-3113

Maes C, Stockmans I, Moermans K et al (2004) Soluble VEGF isoforms are essential for establishing epiphyseal vascularization and regulating chondrocyte development and survival. J Clin Invest 113:188-199

Matsubara H, Hogan DE, Morgan EF et al (2012) Vascular tissues are a primary source of BMP2 expression during bone formation induced by distraction osteogenesis. Bone 51:168-180

Mayr-Wohlfart U, Waltenberger J, Hausser H et al (2002) Vascular endothelial growth factor stimulates chemotactic migration of primary human osteoblasts. Bone 30:472-477

Midy V, Plouet J (1994) Vasculotropin/vascular endothelial growth factor induces differentiation in cultured osteoblasts. Biochem Biophys Res Commun 199:380-386

Nakagawa M, Kaneda T, Arakawa T et al (2000) Vascular endothelial growth factor (VEGF) directly enhances osteoclastic bone resorption and survival of mature osteoclasts. FEBS Lett 473:161-164

Nicosia RF, Nicosia SV, Smith M (1994) Vascular endothelial growth factor, platelet-derived growth factor, and insulin-like growth factor-1 promote rat aortic angiogenesis in vitro. Am J Pathol 145:1023-1029

Niida S, Kaku M, Amano H et al (1999) Vascular endothelial growth factor can substitute for macrophage colony-stimulating factor in the support of osteoclastic bone resorption. J Exp Med 190:293-298

Nuttall ME, Patton AJ, Olivera DL et al (1998) Human trabecular bone cells are able to express both osteoblastic and adipocytic phenotype: implications for osteopenic disorders. J Bone Miner Res 13:371-382

Otomo H, Sakai A, Uchida $\mathrm{S}$ et al (2007) Flt-1 tyrosine kinasedeficient homozygous mice result in decreased trabecular bone volume with reduced osteogenic potential. Bone 40:1494-1501

Park JE, Chen HH, Winer J et al (1994) Placenta growth factor. Potentiation of vascular endothelial growth factor bioactivity, in vitro and in vivo, and high affinity binding to Flt-1 but not to Flk-1/KDR. J Biol Chem 269:25646-25654

Pepper MS, Ferrara N, Orci L et al (1992) Potent synergism between vascular endothelial growth factor and basic fibroblast growth factor in the induction of angiogenesis in vitro. Biochem Biophys Res Commun 189:824-831

Pepper MS, Wasi S, Ferrara N et al (1994) In vitro angiogenic and proteolytic properties of bovine lymphatic endothelial cells. Exp Cell Res 210:298-305

Pittenger MF, Mackay AM, Beck SC et al (1999) Multilineage potential of adult human mesenchymal stem cells. Science 284:143-147

Prockop DJ (2012) New targets for osteoporosis. N Engl J Med 367:2353-2354 
Schipani E, Ryan HE, Didrickson S et al (2001) Hypoxia in cartilage: HIF-1alpha is essential for chondrocyte growth arrest and survival. Genes Dev 15:2865-2876

Shalaby F, Rossant J, Yamaguchi TP et al (1995) Failure of bloodisland formation and vasculogenesis in Flk-1-deficient mice. Nature 376:62-66

Soker S, Takashima S, Miao HQ et al (1998) Neuropilin-1 is expressed by endothelial and tumor cells as an isoform-specific receptor for vascular endothelial growth factor. Cell 92:735-745

Song L, Tuan RS (2004) Transdifferentiation potential of human mesenchymal stem cells derived from bone marrow. FASEB J 18:980-982

Tanaka S, Takahashi N, Udagawa N et al (1993) Macrophage colonystimulating factor is indispensable for both proliferation and differentiation of osteoclast progenitors. $\mathbf{J}$ Clin Invest 91:257-263

Thi MM, Suadicani SO, Spray DC (2010) Fluid flow-induced soluble vascular endothelial growth factor isoforms regulate actin adaptation in osteoblasts. J Biol Chem 285:30931-30941
Verma S, Rajaratnam JH, Denton J et al (2002) Adipocytic proportion of bone marrow is inversely related to bone formation in osteoporosis. J Clin Pathol 55:693-698

Wang Y, Wan C, Deng L et al (2007) The hypoxia-inducible factor alpha pathway couples angiogenesis to osteogenesis during skeletal development. J Clin Invest 117:1616-1626

Yang Q, McHugh KP, Patntirapong S et al (2008) VEGF enhancement of osteoclast survival and bone resorption involves VEGF receptor-2 signaling and beta3-integrin. Matrix Biol 27:589-599

Zelzer E, Olsen BR (2003) The genetic basis for skeletal diseases. Nature 423:343-348

Zelzer E, Olsen BR (2005) Multiple roles of vascular endothelial growth factor (VEGF) in skeletal development, growth, and repair. Curr Top Dev Biol 65:169-187

Zelzer E, McLean W, Ng YS et al (2002) Skeletal defects in $\operatorname{VEGF}(120 / 120)$ mice reveal multiple roles for VEGF in skeletogenesis. Development 129:1893-1904

Zelzer E, Mamluk R, Ferrara N et al (2004) VEGFA is necessary for chondrocyte survival during bone development. Development $131: 2161-2171$ 